\title{
Two Definitions of Superfluid Density
}

\author{
Nikolai V. Prokof'ev and Boris V. Svistunov \\ Russian Research Center "Kurchatov Institute", 123182 Moscow, Russia
}

We point out that two different definitions of the superfluid density - through statistical response to static gauge phase and through dynamic response to altering gauge phase - yield, generally speaking, different quantities in $d<3$. The physics leading to this difference is associated with the equilibrium statistics of supercurrent states. Some experimentally observable consequences of this fact are discussed.

PACS numbers: 67.40.-w, 67.40.Rp, 74.76.-w

In this report we would like to discuss the relation between two alternative definitions of the superfluid density. Consider a system with ring geometry. One way to define the superfluid density is to consider system response to the gauge field generated by the infinitesimal flux $\phi$ threading the ring (we use units such that $\hbar=1$ and particle mass $m=1$ )

$$
\delta \mathcal{F}=\frac{\rho_{S}^{(F)}}{2} \frac{\varphi^{2}}{L^{2-d}},
$$

where $\mathcal{F}$ is the free-energy, $L$ is the system size, $d$ is the system dimensionality (for simplicity we assume that the system size is the same in all directions - other examples are discussed at the end of the paper $)$, and $\varphi=2 \pi\left(\phi / \phi_{0}\right)$ , where $\phi_{0}$ is the flux quantum. We do not specify here what is the value of the flux quantum, since the gauge field considered is not necessarily of elecrtro-magnetic origin. For neutral systems (helium, ultracold atomic gases) it can be introduced by rotating the system. More generally, we may ascribe to the particles fictitious charge and couple it to fictitious gauge field - the idea is to look at the response of the system to the twisted boundary conditions on the ring (we choose axis $\hat{\mathbf{x}}$ as the direction around the ring): $\Psi\left(\ldots, x_{j}=L, \ldots\right)=e^{i \varphi} \Psi\left(\ldots, x_{j}=0, \ldots\right)$ [here $x_{j}$ is the $x$-coordinate of the $j$-th particle]. In fact, this relation may be considered as our initial definition of the phase $\varphi$. The equilibrium particle number current is given then by

$$
J^{(F)}=\rho_{S}^{(F)} \varphi / L
$$

On another hand, one may define superfluid density as a coefficient in effective-long-wavelength action which governs phase fluctuations 1 :

$$
F_{\mathrm{eff}}=\frac{\rho_{S}}{2} \int d V(\nabla \Phi)^{2} .
$$

We do not intend to discuss here under what conditions it is possible to introduce phase field in the long-wavelength limit - this is rather well known and studied problem (see, for example, Refs. 1,21). In most general terms, Eq. (3) works when $\nabla \Phi$ is a well defined operator. In dimensions $d>1$ this would require the existence of the topological long-range order. In $1 \mathrm{D}$, where the concept of the long-range order at any finite temperature is meaningless, Eq. (3) is valid provided the low-energy spectrum of the system is gapless (metallic state) and relaxation times of current states, $1 / \tau$, are very long. In particular, if we label current states in $1 \mathrm{D}$ by index $I$, where

$$
I=\frac{1}{2 \pi} \oint d x \nabla \Phi=\text { integer },
$$

then the sufficient condition reads $1 / \tau_{I} \ll 2 \pi c /(L K)$, where $c$ is the sound velocity, and $K / 2$ is the one-particle density matrix index, $\rho(x) \sim x^{-K / 2}$ at zero temperature. As we discuss below this condition is satisfied at low temperatures, including temperatures exceeding finite-size quantization limit $T \gg 2 \pi c / L$.

Both definitions are used in literature with the unquestioned assumption that $\rho_{S}^{(F)}=\rho_{S}$. In what follows, we demonstrate that although in 3D one indeed may, with macroscopic accuracy, make no distinction between $\rho_{S}^{(F)}$ and $\rho_{S}$, in 2D and especially in $1 \mathrm{D}$, at finite temperature $\rho_{S}^{(F)} \neq \rho_{S}$. We then discuss some interesting consequences associated with this inequality, and how these may be directly tested experimentally.

We start from the well-known relation between $\rho_{S}^{(F)}$ and the statistics of worldline winding numbers, $M$, (see Ref. 3)

$$
\rho_{S}^{(F)}=\frac{T}{L^{d-2}}\left\langle M^{2}\right\rangle
$$

where $M$ is integer. Formally, Eq. (5) immediately follows from the $2 \pi$-periodicity in $\varphi$ of the partition function $Z(\varphi)$, implying that it can be Fourier-expanded as $Z(\varphi)=\sum_{M=-\infty}^{\infty} Z_{M} e^{i M \varphi}$. The statistical average in Eq. (5) is taken with the distribution function $Z_{M}$. The actual extreme usefulness of these formulae for numerical simulations comes from the fact that the numbers $M$ are directly related to the topplogy of the closed particle worldlines in imaginary time 3

On the other hand, the partition function $Z(\varphi)$ can be calculated directly from the effective long-range action (3), employing gauge-invariance argument that external vector-potential and/or twisted boundary condition may be transformed into the phase of the wavefunction. We thus write

$$
Z(\varphi) \propto \int \mathcal{D} \Phi \exp \left\{-F_{\mathrm{eff}}[\Phi]\right\}
$$


where $\Phi$ is parameterized as

$$
\begin{gathered}
\Phi=\Phi_{0}+\frac{x}{L}(2 \pi I-\varphi) \\
\oint d \mathbf{l} \nabla \Phi_{0}=0 \\
\mathcal{D} \Phi \rightarrow \sum_{I=-\infty}^{\infty} \mathcal{D} \Phi_{0} .
\end{gathered}
$$

We then immediately see that

$$
Z(\varphi)=Z_{0} \sum_{I=-\infty}^{\infty} \exp \left\{-\frac{\rho_{S} L^{d-2}}{2 T}(2 \pi I-\varphi)^{2}\right\}
$$

where $Z_{0}$ is independent of $\varphi$.

The second derivative of $-T \ln Z(\varphi)$ at $\varphi=0$, related to $\rho_{S}^{(F)} L^{d-2}$, then yields

$$
\rho_{S}^{(F)}=\rho_{S}\left(1-\frac{4 \pi^{2} \rho_{S} L^{d-2}}{T}\left\langle I^{2}\right\rangle\right),
$$

the averaging being performed with the normalized distribution

$$
W_{I} \propto \exp \left\{-\frac{2 \pi^{2} \rho_{S} L^{d-2}}{T} I^{2}\right\} .
$$

Eqs. (11) and (12) establish a relation between $\rho_{S}^{(F)}$ and $\rho_{S}$. One can also directly relate $\rho_{S}$ to the statistics of worldline winding numbers. Using the known properties of $\theta_{3}$-functions, the following identity is valid

$$
\begin{aligned}
Z(\varphi) & \propto \sum_{I=-\infty}^{\infty} \exp \left\{-\frac{\rho_{S} L^{d-2}}{2 T}(2 \pi I-\varphi)^{2}\right\} \\
& \equiv \sum_{M=-\infty}^{\infty} \exp \left\{-\frac{T}{2 \rho_{S} L^{d-2}} M^{2}-i M \varphi\right\}
\end{aligned}
$$

where $M$ 's are some integers, which, by the uniqueness of the Fourier transform, are immediately identified with the worldline winding numbers. We thus see that the worldline winding numbers have Gaussian distribution

$$
Z_{M} \propto \exp \left\{-\frac{T}{2 \rho_{S} L^{d-2}} M^{2}\right\} .
$$

As is clear from Eq. 11], $\rho_{S}^{(F)}$ and $\rho_{S}$ in general differ. However, at $T \rightarrow 0$ and finite $L$, i.e. in the "mesoscopic" limit, we find that $\rho_{S}^{(F)}=\rho_{S}$. Moreover, in 3D case, one may safely replace $\rho_{S}^{(F)}$ with $\rho_{S}$ since, according to Eqs. (11 12), finite-size corrections are exponentially small in macroscopic parameter: the probability of finding non-zero $I= \pm 1$ dies away as $\exp \left\{-2 \pi^{2} \rho_{S} L / T\right\}$.
This is probably the reason why it became a custom to identify $\rho_{S}^{(F)}$ and $\rho_{S}$.

In $1 \mathrm{D}$ systems the situation changes drastically. In the thermodynamic limit, when $L \rightarrow \infty$ first, that is $T L \rightarrow \infty$ at any finite temperature, we have negligible $\rho_{S}^{(F)}\left[\sim L T \exp \left\{-L T / 2 \rho_{S}\right\}\right.$, see Eq. (14)] while $\rho_{S}$ is finite. At this point we would like to emphasize that zero $\rho_{S}^{(F)}$ is not in contradiction with the fact that the system may remain superfluid in any dynamic sense. Equations (11 12) make it clear that $\rho_{S}^{(F)} \rightarrow 0$ simply due to the broad distribution over the current states, with typical $I \sim(L T)^{1 / 2} \gg 1$. However, relaxation times of current states in $1 \mathrm{D}$ may be very long 4 (see also below), up to astronomical scale. Therefore, out-of-equilibrium state obtained experimentally, say, by switching on/off the gauge phase $\varphi$, will support a supercurrent $j \sim \rho_{S} \varphi / L$.

To estimate relaxation times $\tau_{I}$ in $1 \mathrm{D}$ rings (obviously, for the relaxation time to be finite the translational invariance must be violated by impurities, or lattice potential) one may use an effective Hamiltonian describing low energy dynamics of supercurrent states and density fluctuations 1 . Relaxation rate is obtained then from the golden-rule expression for the terms breaking translational invariance. Formally, this problem is identical to the study of particle dynamics with Ohmic coupling to the oscillator bath environment 5 - an analogy widely used in 1D systems since Ref. 6. The standard result for the transition rate from $I$ to $I+1$ due to single impurity backscattering potential, $\Delta$, is given by (see, e.g., Ref. 母)

$$
\tau_{I}^{-1} \sim \frac{\Delta^{2}}{\omega_{o}}\left(\frac{T}{\omega_{o}}\right)^{2 / K-1}
$$

where $\omega_{o}$ is some model-dependent high-energy cut-off. If parameter $K$ is small, then at low temperature one may easily get extremely long $\tau_{I}$. The answer for the disordered ring with white-noise disorder is just the single impurity expression (15) multiplied by $L$.

One may directly test the striking difference between $\rho_{S}^{(F)}$ and $\rho_{S}$ in $1 \mathrm{D}$ systems experimentally. In fact, we propose a unique measuring device for magnetic fields, free of limitations set by quantization of magnetic flux. Suppose we would like to measure local magnetic field at the point $\mathbf{R}$ by placing at this point a device recording magnetic flux through some small surface with subsequent reading the information recorded. We point out that such a device can be realized as an ensemble of 1D (quasi-)superconducting rings. Being placed at the point $\mathbf{R}$ at high temperature (say, $T \sim \omega_{0}$ ) when relaxation times are short, and then cooled down to low temperatures when relaxation times become very long (much longer than expected "storage time"), the ensemble will not respond to the local field, since in this case the response is statistical and $\rho_{S}^{(F)} \approx 0$. However, removing cooled device from the point $\mathbf{R}$ we excite finite supercurrent, since now we probe dynamic response $\rho_{S}$. It is important to note, that, in contrast to the case of mas- 
sive superconducting ring, the stored magnetic flux is not quantized.

Another interesting experiment directly follows from Eq. (12). A single 1D ring being cooled down to low temperature will typically end up in a state with non-zero supercurrent $j \sim\left(\rho_{S} T_{\text {ex }} / L\right)^{1 / 2}$, where $T_{\text {ex }}$ is the "freezing" temperature at which the current relaxation time becomes comparable to the experimental cooling time.

The $2 \mathrm{D}$ case is even more intriguing. For an isotropic system with $L_{x}=L_{y}$, Eqs. (11), (12) and (14) are system-size independent, in particular

$$
\begin{aligned}
& \rho_{S}^{(F)}=\rho_{S}\left(1-\frac{4 \pi^{2} \rho_{S}}{T}\left\langle I^{2}\right\rangle\right), \\
& W_{I} \propto \exp \left\{-\frac{2 \pi^{2} \rho_{S}}{T} I^{2}\right\} .
\end{aligned}
$$

The famous Nelson-Kosterlitz formula

$$
T_{c}=\frac{\pi}{2} \rho_{S}
$$

relates transition temperature and $\rho_{S}$ [not $\rho_{S}^{(F)}$ !] (see Ref. 8, and also Ref. 2). Substituting it into Eq. (16), we find that with very high accuracy

$$
\rho_{S}^{(F)}\left(T_{c}\right)=\rho_{S}\left(1-16 \pi e^{-4 \pi}\right) .
$$

For purely numerical reasons the relative difference $\left(\rho_{S}^{(F)}-\rho_{S}\right) / \rho_{S}<2 \cdot 10^{-4}$ is very small. That is why in a recent state-of-the-art numerical study of the BerezinskiiKosterlitz-Thouless transition $\theta$ formally incorrect relation $T_{c}=(\pi / 2) \rho_{S}^{(F)}$ (or $\left.\left\langle M^{2}\right\rangle=2 / \pi^{3}\right)$ was successfully used to fit the data. However, unlike in $3 \mathrm{D}$ case, the difference between $\rho_{S}^{(F)}$ and $\rho_{S}$ is not controlled any more by small parameters. Furthermore, if a $2 \mathrm{D}$ system is elongated geometrically: $L_{x}>L_{y}$, then

$$
\begin{aligned}
\rho_{S, x}^{(F)} & =\rho_{S}\left(1-\frac{4 \pi^{2} \rho_{S} L_{y}}{T L_{x}}\left\langle I^{2}\right\rangle\right), \\
W_{I, x} & \propto \exp \left\{-\frac{2 \pi^{2} \rho_{S} L_{y}}{T L_{x}} I^{2}\right\},
\end{aligned}
$$

where index $x$ for $\rho_{S}^{(F)}$ and $W_{I}$ is used to specify that we are dealing with the response to the twisted boundary condition along $x$-axis. Clearly, at $L_{x} \gg L_{y}$ the physics becomes analogous to that of $1 \mathrm{D}$ rings. We thus see that in $2 \mathrm{D} \rho_{S}^{F}$ depends on the sample geometry and at $L_{x} \geq$ $4 \pi L_{y}$, the value of $\rho_{S}^{(F)}$ is not related to $T_{c}$, Eq. (17), even approximately.

It seems rather easy experimentally to confirm these conclusions by producing narrow Carbino-disk samples. Since in this case we are dealing with macroscopic phase transition phenomenon, at $T<T_{c}$ the current relaxation times become astronomically long and the abovementioned experiments on $1 \mathrm{D}$ rings would simply require cooling below $T_{c}$.
We are grateful to Yu. Kagan for his interest and discussions. This work was supported by the Russian Foundation for Basic Research (under Grant No. 98-02-16262) and by the Grant INTAS-97-0972 [of the European Community].

${ }^{1}$ V.N. Popov, Functional Integrals in Quantum Field Theory and Statistical Physics, Dordrecht: Reidel (1983).

2 B.V. Svistunov, J. Moscow Phys. Soc. 2, 283 (1992).

${ }^{3}$ E.L. Pollock and D.M. Ceperley, Phys. Rev. B 36, 8343 (1987)

${ }^{4}$ V.A. Kashurnikov, A.I. Podlivaev, N.V. Prokof'ev, and B.V. Svistunov, Phys. Rev. B 53, 13091 (1996).

${ }^{5}$ A.O. Caldeira and A.J. Leggett, Ann. Phys. 149, 374 (1983).

${ }^{6}$ C.L. Kane and M.P.A. Fisher, Phys. Rev. Lett. 68, 1220 (1992).

7 A.J. Leggett, S. Chakravarty, A.T. Dorsey, M.P.A. Fisher, A. Garg, and W. Zwerger, Rev. Mod. Phys. 59, 1 (1987); Quantum Tunneling in Condensed Media, eds. Yu. Kagan and A.J. Leggett, North-Holland, Elsevier (1992).

${ }^{8}$ D.R. Nelson and J.M. Kosterlitz, Phys. Rev. Lett. 391201 (1977).

${ }^{9}$ K. Harada and N. Kawashima, J. Phys. Soc. Jpn. 67, 2768 (1998). 